\title{
Application of Laplace Iteration method to Study of Nonlinear Vibration of laminated composite plates
}

\section{Abstract}

In this paper, free vibration characteristics of laminated composite plates are investigated. A model is developed for a composite layer based on the consideration of non-linear terms in von-Karman's non-linear deformation theory. The governing partial equation of motion is reduced to an ordinary non-linear equation and then solved using LIM method. The variation of frequency ratio of the Isotropic and composite plates is brought out considering parameters such as aspect ratio, fiber arrangements (orientation), number of layers, and modal ratios.

\section{Keywords}

Nonlinear analytical analysis; Nonlinear Vibration; Composite

Plate, Classical Plate Theory; Laplace Iteration Method

\author{
H. Rafieipour ${ }^{*}$, A. Lotfavar, \\ A. Masroori, E. Mahmoodi \\ Department of Mechanical and Aerospace \\ Engineering, Shiraz University of Technology, \\ 71555-313 Shiraz, Iran \\ Received 01 Nov 2012 \\ In revised form 14 Dec 2012 \\ * Author email: H.Rafieipour@sutech.ac.ir \\ Lotfavar@sutech.ac.ir
}

\section{INTRODUCTION}

Laminated composite plates, due to their high specific strength and stiffness have been increasingly used in a wide range of aerospace, mechanical, and civil applications. By tailoring the sequence of the stacks and the thickness of the layers, composite laminates' characteristics can be matched to the structural requirements with no difficulty. To use composite laminates efficiently, an accurate knowledge of vibration characteristics is essential. Vibration not only creates excessive noise and wastes energy but also may result in catastrophic failures. These phenomena, when the system operates around its natural frequencies, would be even more disastrous.

Many publications have dealt with the linear vibrations of laminated composites. In these cases, the equation of motion is obtained easily and then by a reduction into a generalized eigenvalue problem, frequencies and mode shapes are determined. However, in many working conditions, plates are subjected to large amplitudes, so a nonlinear frequency analysis is required as a result. Obtaining an exact solution for the nonlinear free vibration of composite plates is very difficult due to the complexity of the equation of motion. The first approximate solution developed by Chu and Her$\operatorname{man}[1]$ in 1956, was the start point for so many other numerical methods introduced in the following years such as the finite element method (FEM)[2], the discrete singular convolution method (DSC), the strip element method, and the Ritz methods[3]. 
Singh et al.[4] used direct numerical integration to study non-linear vibration of rectangular laminated composite plates in 1990. Using Kirchhoff hypothesizes and von-Karman strain-stress relations, they derived governing equations. They also employed harmonic oscillating assumptions and investigated large amplitude vibrations for various arrangements.

Singha and Ganapathi[5], examined thin isotropic composite plates exploiting finite element method. They also considered the effects of shear deformation, rotational inertia and in plane inertia, in their formulation. In their study, non-linear matrices derived from Lagrange equations were solved using direct iteration technique and also they examined variations of non-linear frequency ratio versus various amplitudes considering influencing parameters, such as boundary conditions and fiber orientations.

Malekzadeh and Karami[6], investigated non-linear vibrations of composite plates considering first order shear deformation theory employing Quadrature method. Effects of various parameters, such as foundations, thickness to length ratio and aspect ratio on frequency ratio were also examined in their study.

Although numerical approaches are applicable to a wide range of practical cases, approximate analytical methods provide highly accurate solutions and a deep physical insight. One of the main approximate analytical approaches on nonlinear vibration analysis is Perturbation Method. This method is effective just in solving weakly nonlinear differential equations. Because of the limited application of the perturbation methods, newer approaches have been developed during recent years which are more powerful. For example, Pirbodaghi et al.[7] used the homotopy analysis method (HAM) to obtain an approximate analytical solution for nonlinear vibrations of thin laminated composite plates and showed effectiveness of this method to obtain an accurate solution with relatively less computational effort. Moreover, the energy balance method (EBM) [8], the max-min approach (MMA) [9], the homotopy perturbation method (HPM) [10] and so many others have been proved to be strong approximate analytical approaches, obtaining accurate results for nonlinear differential equations [11].

The LIM method, which was introduced by Rafieipour et al.[12] is a very powerful method in solving non-linear differential equations and its effectiveness is proved in studying non-linear vibration of composite beams. It was shown that this is one of the best analytical methods due to the rate of convergence and its accuracy.

In the present paper, LIM method is used to obtain approximate analytical solutions for nonlinear vibrations of a thin laminated composite plate. A variational method is employed to derive the governing equations. The nonlinear term added is associated with von-Karman strain displacement relation. LIM method is used to achieve nonlinear natural frequency and its excellent accuracy for the wide range of amplitude values is satisfied. A comparison with results in other articles has been done to validate the answers. The impact of layer arrangements and aspect ratio effects on the free vibration are investigated in detail.

\section{FORMULATION}

Consider a thin laminated composite plate of length $a$, width $b$, and thickness $h$ as shown in figure 1 . A positive set of coordinate system demonstrated in a way that reference surface is taken as the 
mid plane and its origin is considered at the corner of the plate. The plate is supposed to be simply supported along all its edges. In addition, no slip condition between the layers is assumed.

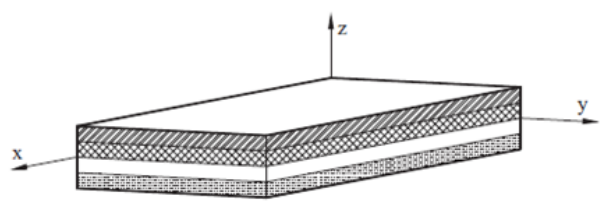

Figure 1 Schematic of the laminated composite plate

Assume $u, v$ and $w$ are the displacements of an arbitrary point of the plane in $x, y$ and $z$ axis, $u_{0}$ and $v_{0}$ to be the corresponding displacements of that point in the mid-plane and $\varepsilon^{0}$ and $\kappa$ to be the mid-plain strain and mid-plane curvature respectively; mechanical shear relation can be demonstrated as[13]:

$$
\left\{\begin{array}{c}
\varepsilon_{x} \\
\varepsilon_{y} \\
\varepsilon_{x y}
\end{array}\right\}=\left\{\begin{array}{c}
\varepsilon_{x}^{0} \\
\varepsilon_{y}^{0} \\
\varepsilon_{x y}^{0}
\end{array}\right\}+z\left\{\begin{array}{c}
\kappa_{x} \\
\kappa_{y} \\
\kappa_{x y}
\end{array}\right\}
$$

Following von-Karman's strain-displacement assumptions the in plane strain, shear strain and plane curvatures can be expressed as:

$$
\begin{gathered}
\left\{\begin{array}{c}
\varepsilon_{x}^{0} \\
\varepsilon_{y}^{0} \\
\varepsilon_{x y}^{0}
\end{array}\right\}=\left\{\begin{array}{c}
\frac{\partial u_{0}}{\partial x}+\frac{1}{2}\left(\frac{\partial w_{0}}{\partial x}\right)^{2} \\
\frac{\partial v_{0}}{\partial y}+\frac{1}{2}\left(\frac{\partial w_{0}}{\partial y}\right)^{2} \\
\left(\frac{\partial u_{0}}{\partial y}+\frac{\partial v_{0}}{\partial x}+\frac{\partial w_{0}}{\partial x} \frac{\partial w_{0}}{\partial y}\right)
\end{array}\right\} \\
\left\{\begin{array}{c}
\kappa_{x} \\
\kappa_{y} \\
\kappa_{x y}
\end{array}\right\}=\left\{\begin{array}{c}
-\frac{\partial^{2} w_{0}}{\partial x^{2}} \\
-\frac{\partial w_{0}}{\partial y} \\
-2 \frac{\partial^{2} w_{0}}{\partial x \partial y}
\end{array}\right\}
\end{gathered}
$$


According to Classical Theory of Elasticity, the strain-stress relations for each layer can be derived as:

$$
\left\{\begin{array}{c}
\sigma_{1} \\
\sigma_{2} \\
\sigma_{6}
\end{array}\right\}_{k}=\left(\begin{array}{ccc}
Q_{11} & Q_{12} & 0 \\
Q_{12} & Q_{22} & 0 \\
0 & 0 & Q_{66}
\end{array}\right)_{k}\left\{\begin{array}{c}
\varepsilon_{1} \\
\varepsilon_{2} \\
\varepsilon_{6}
\end{array}\right\}_{k}
$$

Where the numbers $1,2,6$ referred to principal axis of each layer. $\left(Q_{i j}\right)_{k}(i=1,2,6)$ are the coefficients of the reduced stiffness matrix at the $k$ th layer and are defined as:

$$
\begin{array}{ll}
Q_{11}=\frac{E_{11}}{1-v_{12} v_{21}} \quad, & Q_{12}=\frac{v_{12} E_{22}}{1-v_{12} v_{21}}=\frac{v_{21} E_{11}}{1-v_{12} v_{21}} \\
Q_{22}=\frac{E_{22}}{1-v_{12} v_{21}} \quad, \quad Q_{66} & =G_{12}
\end{array}
$$

Below stress-strain relations are obtained from the axis transformation of the each layer stressstrain equations referred to global axes:

$$
\left\{\begin{array}{c}
\sigma_{x} \\
\sigma_{y} \\
\sigma_{x y}
\end{array}\right\}_{k}=\left[\begin{array}{lll}
\bar{Q}_{11} & \bar{Q}_{12} & \bar{Q}_{16} \\
\bar{Q}_{12} & \bar{Q}_{22} & \bar{Q}_{26} \\
\bar{Q}_{16} & \bar{Q}_{26} & \bar{Q}_{66}
\end{array}\right]_{k}\left(\left\{\begin{array}{c}
\varepsilon_{x}^{0} \\
\varepsilon_{y}^{0} \\
\varepsilon_{x y}^{0}
\end{array}\right\}+z\left\{\begin{array}{c}
\kappa_{x} \\
\kappa_{y} \\
\kappa_{x y}
\end{array}\right\}\right)_{k}
$$

And $\left(Q_{i j}\right)_{k}(i=1,2,6)$ are plane stress-reduced stiffness coefficients.

Constitutive equations which relate force and moment resultants to the strains through an appropriate integration along the thickness can be developed as:

$$
\left(\begin{array}{l}
N_{x} \\
N_{y} \\
N_{x y}
\end{array}\right)=\left(\begin{array}{ccc}
A_{11} & A_{12} & A_{16} \\
A_{12} & A_{22} & A_{26} \\
A_{16} & A_{26} & A_{66}
\end{array}\right)\left\{\begin{array}{c}
\varepsilon_{x}^{0} \\
\varepsilon_{y}^{0} \\
\varepsilon_{x y}^{0}
\end{array}\right\}+\left(\begin{array}{ccc}
B_{11} & B_{12} & B_{16} \\
B_{12} & B_{22} & B_{26} \\
B_{16} & B_{26} & B_{66}
\end{array}\right)\left\{\begin{array}{c}
\kappa_{x} \\
\kappa_{y} \\
\kappa_{x y}
\end{array}\right\}
$$




$$
\left(\begin{array}{l}
M_{x} \\
M_{y} \\
M_{x y}
\end{array}\right)=\left(\begin{array}{lll}
B_{11} & B_{12} & B_{16} \\
B_{12} & B_{22} & B_{26} \\
B_{16} & B_{26} & B_{66}
\end{array}\right)\left\{\begin{array}{c}
\varepsilon_{x}^{0} \\
\varepsilon_{y}^{0} \\
\varepsilon_{x y}^{0}
\end{array}\right\}+\left(\begin{array}{ccc}
D_{11} & D_{12} & D_{16} \\
D_{12} & D_{22} & D_{26} \\
D_{16} & D_{26} & D_{66}
\end{array}\right)\left\{\begin{array}{c}
\kappa_{x} \\
\kappa_{y} \\
\kappa_{x y}
\end{array}\right\}
$$

Where,

$$
\begin{aligned}
& A_{i j}=\sum_{k=1}^{n}\left(\bar{Q}_{i j}\right)_{k}\left(h_{k}-h_{k-1}\right) \\
& B_{i j}=\frac{1}{2} \sum_{k=1}^{n}\left(\bar{Q}_{i j}\right)_{k}\left(h_{k}^{2}-h_{k-1}^{2}\right) \\
& D_{i j}=\frac{1}{3} \sum_{k=1}^{n}\left(\bar{Q}_{i j}\right)_{k}\left(h_{k}^{3}-h_{k-1}^{3}\right)
\end{aligned}
$$

$\mathrm{A}_{\mathrm{ij}}, \mathrm{B}_{\mathrm{ij}}$, and $\mathrm{D}_{\mathrm{ij}}$ are called extensional stiffnesses, bending-extensional coupling stiffnesses and bending stiffness, respectively. The equations of motion are derived from Hamilton's principle as follows[14]:

$$
0=\delta \int_{t_{1}}^{t_{2}}(U-T) d \tilde{t}
$$

Where $U$, the potential energy of the plate and $T$, the kinematic energy of the plate are given by:

$$
\begin{gathered}
U=\frac{1}{2} \int_{V}\left(\sigma_{x x} \varepsilon_{x x}+\sigma_{y y} \varepsilon_{y y}+2 \sigma_{x y} \varepsilon_{x y}\right) d z d x d y \\
T=\frac{1}{2} \int_{0}^{a} \int_{0}^{b}\left(\sum \rho_{i} h_{i}\right) \dot{w}^{2} d x d y
\end{gathered}
$$

Substituting Eqs.(11) and (12) into the Hamilton's equation of motion and with an appropriate integration along the thickness and then by equating $\delta u_{0}, \delta v_{0}$, and $\delta w_{0}$ coefficients to zero, one obtains the below relations: 


$$
\begin{gathered}
\frac{\partial N_{x}}{\partial x}+\frac{\partial N_{x y}}{\partial y}=0 \\
\frac{\partial N_{y}}{\partial y}+\frac{\partial N_{x y}}{\partial x}=0 \\
\frac{\partial^{2} M_{x}}{\partial x^{2}}+2 \frac{\partial^{2} M_{x y}}{\partial x \partial y}+\frac{\partial^{2} M_{y}}{\partial y^{2}}+\frac{\partial}{\partial x}\left(N_{x} \frac{\partial w}{\partial x}\right)+\frac{\partial}{\partial x}\left(N_{x y} \frac{\partial w}{\partial y}\right)+\frac{\partial}{\partial y}\left(N_{x y} \frac{\partial w}{\partial x}\right) \\
+\frac{\partial}{\partial y}\left(N_{y} \frac{\partial w}{\partial y}\right)=\rho h \frac{\partial^{2} w}{\partial t^{2}}
\end{gathered}
$$

By assuming simply supported boundary condition, the relations below are considered for displacement equations to satisfy the boundary conditions:

$$
\begin{aligned}
& u=U(t) \operatorname{Sin} \frac{2 m \pi x}{a} \operatorname{Sin} \frac{n \pi y}{b} \\
& v=V(t) \operatorname{Sin} \frac{m \pi x}{a} \operatorname{Sin} \frac{2 n \pi y}{b} \\
& w=W(t) \operatorname{Sin} \frac{m \pi x}{a} \operatorname{Sin} \frac{n \pi y}{b}
\end{aligned}
$$

And $U(t), V(t)$, and $W(t)$ are the maximum displacements of plate center point along principal axes $x, y$, and $z$ respectively. $U(t)$ and $V(t)$ can be expressed in terms of $W(t)$ using first two equations of Eq. (13) and then by employing Galerkin method and substituting $U(t)$ and $V(t)$ in terms of $W(t)$ into Eq. (13), the governing equation can be written as:

$$
\frac{d^{2} W(t)}{d t^{2}}+\alpha_{1} W(t)+\alpha_{2} V^{2}(t)+\alpha_{3} W^{3}(t)=0
$$

Supposing $W_{\max }$ as the maximum vibration amplitude of the plate center, the initial conditions of the center of the plate can be expressed as:

$$
W(0)=W_{\max } \quad, \quad \frac{d W(0)}{d t}=0
$$

\section{DESCRIPTION OF THE PROPOSED METHOD}

Using the Laplace Transformation method, an analytical approximated technique is proposed to present an accurate solution for nonlinear differential equations. To clarify the basic ideas of proposed method consider the following second order differential equation, 


$$
\ddot{u}(t)+N\{u(t)\}=0
$$

with artificial zero initial conditions and $N$ is a nonlinear operator. Adding and subtracting the term $\omega^{2} u(t)$, the Eq. (17) can be written in the form

$$
\ddot{u}(t)+\omega^{2} u(t)=L\{u(t)\}=f(u(t))
$$

where $L$ is a linear operator and

$$
f(u(t))=\omega^{2} u(t)-N\{u(t)\}
$$

Taking Laplace transform of both sides of the Eq. (18) in the usual way and using the homogenous initial conditions gives

$$
\left(s^{2}+\omega^{2}\right) U(s)=\mathfrak{I}\{f(u(t))\}
$$

where $s$ and $\mathfrak{I}$ are the Laplace variable and operator, correspondingly. Therefore it is obvious that

$$
U(s)=\mathfrak{I}\{f(u(t))\} G(s)
$$

where

$$
G(s)=\frac{1}{s^{2}+\omega^{2}}
$$

Now, implementing the Laplace inverse transform of Eq. (21) and using the Convolution theorem offer

$$
u(t)=\int_{0}^{t} f(u(\tau)) g(t-\tau) d \tau
$$

where

$$
g(t)=\mathfrak{I}^{-1}\{G(s)\}=\frac{1}{\omega} \sin (\omega t)
$$

Substituting Eq. (19) and (24) into (23) gives 


$$
u(t)=\int_{0}^{t}\left(\omega^{2} u(\tau)-N\{u(\tau)\}\right) \frac{1}{\omega} \sin (\omega(t-\tau)) d \tau
$$

Now, the actual initial conditions must be imposed. Finally the following iteration formulation can be used [15]

$$
u_{n+1}=u_{0}+\frac{1}{\omega} \int_{0}^{t}\left(\omega^{2} u_{n}(\tau)-N\left\{u_{n}(\tau)\right\}\right) \sin (\omega(t-\tau)) d \tau
$$

Knowing the initial approximation $u_{0}$, the next approximations $u_{n}, n>0$ can be determined from previous iterations. Consequently, the exact solution may be obtained by using:

$$
u=\lim _{n \rightarrow \infty} u_{n}
$$

The method proposed here can be applied in various non-linear problems. However, there is no need for any linearization and neither any small parameter. Also, the obtained approximate solutions converge quickly to the exact one.

\section{IMPLEMENTATION OF THE PROPOSED METHOD}

Rewriting $\mathrm{Eq}(15)$ in the standard form of Eq. (18) results in the following equation:

$$
\frac{d^{2} \eta(t)}{d t^{2}}+\omega^{2} \eta(t)=f(\eta(t))
$$

where

$$
\begin{aligned}
& f(\eta(t))=\omega^{2} \eta(t)-N\{\eta(t)\} \\
& N\{\eta(t)\}=\alpha_{1} \eta(t)+\alpha_{2} \eta^{2}(t)+\alpha_{3} \eta^{3}(t),
\end{aligned}
$$

Applying the proposed method, the following iterative formula is formed as:

$$
\eta_{n+1}(t)=\eta_{0}(t)+\frac{1}{\omega} \int_{0}^{t} f\left(\eta_{n}(\tau)\right) \sin (\omega(t-\tau)) d \tau
$$

Eq. (28) will be homogeneous, when $f(\eta(t))$ has a value of zero. So, its homogeneous.

Solution

$$
\eta_{0}(t)=A \cos (\omega t)
$$


is considered as the zero approximation for using in iterative Eq.(30).

Expanding $f\left(\eta_{0}(\tau)\right)$ we have:

$$
f\left(\eta_{0}(\tau)\right)=\left(-\alpha_{1} A+\omega^{2} A-\frac{3}{4} \alpha_{3} A^{3}\right) \cos (\omega t)-\frac{1}{4} \alpha_{3} A^{3} \cos (3 \omega t)-\frac{1}{2} \alpha_{2} A^{2}(1+\cos (2 \omega t))
$$

Considering the relation:

$$
\frac{1}{\omega} \int_{0}^{t}(\cos (m \omega \tau)) \sin (\omega(t-\tau)) d \tau= \begin{cases}\frac{\cos (\omega t)-\cos (m \omega t)}{\omega^{2}\left(m^{2}-1\right)} & m \neq 1 \\ \frac{t \sin (\omega t)}{2 \omega} & m=1\end{cases}
$$

The coefficient of the term $\cos (\omega t)$ in $f\left(\eta_{0}(\tau)\right)$ should be vanished in order to avoid secular terms in subsequent iterations.

As a result, the first approximation of the frequency can be expressed as:

$$
\omega=\sqrt{\alpha_{1}+\frac{3}{4} \alpha_{3} A^{2}}
$$

Substituting Eq. (31) into (30) and eliminating the secular terms which is the coefficient of $\cos (\omega t)$ in forcing function $f(\eta)$, results in:

$$
\begin{aligned}
& \eta_{1}(t)=\frac{1}{96 \omega^{2}}\left\{\left(32 \alpha_{2} A^{2}+96 \omega^{2} A-3 \alpha_{3} A^{3}\right) \cos (\omega t)+16 \alpha_{2} A^{2} \cos (2 \omega t)\right. \\
&\left.+3 \alpha_{3} A^{3} \cos (3 \omega t)-48 \alpha_{2} A^{2}\right\}
\end{aligned}
$$

This is the first approximation of $\eta(t)$. Replacement of Eq. (35) in Eq. (30) and applying the procedure again results in the second approximation of $\eta(t)$ as

$$
\eta_{2}(t)=\frac{1}{1981808640} \frac{1}{A^{3}}\left(\begin{array}{l}
\mathrm{I}_{0}+\mathrm{I}_{1} \cos (\omega t)+\mathrm{I}_{2} \cos (2 \omega t)+\mathrm{I}_{3} \cos (3 \omega t) \\
+\mathrm{I}_{4} \cos (4 \omega t)+\mathrm{I}_{5} \cos (5 \omega t)+\mathrm{I}_{6} \cos (6 \omega t) \\
+\mathrm{I}_{7} \cos (7 \omega t)+\mathrm{I}_{8} \cos (8 \omega t)+\mathrm{I}_{9} \cos (9 \omega t)
\end{array}\right)
$$


Where $\mathrm{I}_{i}$ are given in Appendix.

The coefficient of $\cos (\omega t)$ in forcing function assumed to be zero in order to evade the secular terms:

$$
\omega^{8}+\beta_{6} \omega^{6}+\beta_{4} \omega^{4}+\beta_{2} \omega^{2}+\beta_{0}=0
$$

where

$$
\begin{aligned}
& \beta_{6}=\left(-\frac{5^{2}}{2^{5}} \alpha_{3} A^{2}+\frac{1}{3} \alpha_{2} A-\alpha_{1}\right) \\
& \beta_{4}=\left(\frac{3}{2^{6}} \alpha_{3}^{2} A^{4}-\frac{3}{2^{2}} \alpha_{2} \alpha_{3} A^{3}+\left(\frac{1}{2^{5}} \alpha_{1} \alpha_{3}+\frac{5}{2 \cdot 3} \alpha_{2}^{2}\right) A^{2}-\frac{1}{3} \alpha_{1} \alpha_{2} A\right) \\
& \beta_{2}=\left(-\frac{3^{2}}{2^{12}} \alpha_{3}^{3} A^{6}+\frac{1}{2^{5}} \alpha_{2} \alpha_{3}^{2} A^{5}+\frac{5}{2 \cdot 3^{2}} \alpha_{2}^{3} A^{3}-\frac{79}{2^{5} \cdot 3} \alpha_{2}^{2} \alpha_{3} A^{2}\right) \\
& \beta_{0}=\left(\frac{3}{2^{16}} \alpha_{3}^{4} A^{8}-\frac{3}{2^{12}} \alpha_{2} \alpha_{3}^{3} A^{7}+\frac{3 \cdot 5}{2^{9}} \alpha_{2}^{2} \alpha_{3}^{2} A^{6}-\frac{5}{2^{3} \cdot 3}\right)
\end{aligned}
$$

Solving Eq. (37) estimates the value of $\omega$ for the actual natural frequency of the system.

\section{RESULTS AND DISCUSSIONS}

In order to verify the precision of the suggested method, current results were compared with other articles.

\begin{tabular}{|c|c|c|c|c|c|c|}
\hline \multirow[t]{2}{*}{$W / h$} & \multicolumn{3}{|c|}{$(a / b=1)$} & \multicolumn{3}{|c|}{$(a / b=2)$} \\
\hline & LIM & HAM & $\operatorname{Ref}[4]$ & LIM & HAM & $\overline{R e f}[4]$ \\
\hline 0.2 & 1.0251 & 1.0252 & 1.0208 & 1.0285 & 1.0285 & 1.0254 \\
\hline 0.4 & 1.0967 & 1.0967 & 1.0809 & 1.1091 & 1.1091 & 1.0982 \\
\hline 0.6 & 1.2056 & 1.2056 & 1.1743 & 1.2307 & 1.2306 & 1.2097 \\
\hline 0.8 & 1.3423 & 1.3422 & 1.2937 & 1.3819 & 1.3818 & 1.3505 \\
\hline 1 & 1.4991 & 1.4988 & 1.4327 & 1.5541 & 1.5537 & 1.5124 \\
\hline 1.2 & 1.6703 & 1.6698 & --- & 1.741 & 1.7404 & --- \\
\hline 1.4 & 1.852 & 1.8512 & 1.7503 & 1.9384 & 1.9376 & 1.877 \\
\hline 1.6 & 2.0412 & 2.0404 & --- & 2.1435 & 2.1425 & --- \\
\hline 1.8 & 2.2364 & 2.2353 & --- & 2.3543 & 2.353 & --- \\
\hline 2 & 2.436 & 2.4347 & 2.2828 & 2.5694 & 2.5679 & 2.4798 \\
\hline
\end{tabular}

Table 1 illustrates the frequency ratio of square and rectangular plates. It is observable that our results are in excellent agreement with the results provided by other references.

It can be observed that the second displacement coefficient is zero for square plates.

Table 1 Frequency ratio of isotropic rectangular and square plates using various methods $(v=0.3)$

Latin American Journal of Solids and Structures 10(2013) $781-795$ 
In both cases, the difference between the non-linear frequency and linear frequency increased by increasing the amplitude of vibration. This difference increased even more when aspect ratio increased (figure 2).

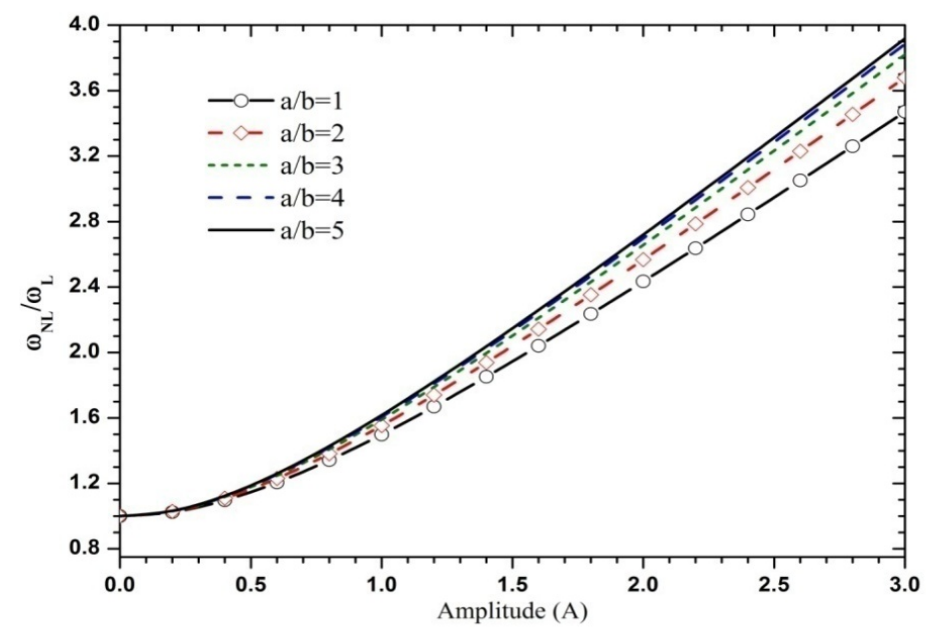

Figure 2 The effect of aspect ratio on the nonlinear to the linear frequency ratio

In table 2, the Variation of frequency ratios versus non-dimensional amplitude ratio for symmetrical and non-symmetrical square plate arrangements are shown. The symmetrical arrangement plates have the same frequency ratios values.

Table 2 Frequency ratio of composite square plate with different arrangements using various methods $\left(E_{1} / E_{2}=40, G_{12} / E_{2}=0.5, v_{12}=0.25\right)$

\begin{tabular}{|c|c|c|c|c|c|c|c|c|c|}
\hline \multirow[t]{2}{*}{$W / h$} & \multicolumn{3}{|c|}{$\left[0^{\circ} / 90^{\circ} / 0^{\circ} / 90^{\circ}\right]$} & \multicolumn{3}{|c|}{$\left[0^{\circ} / 90^{\circ} / 90^{\circ} / 0^{\circ}\right]$} & \multicolumn{3}{|c|}{$\left[90^{\circ} / 0^{\circ} / 0^{\circ} / 90^{\circ}\right]$} \\
\hline & LIM & HAM & $\operatorname{Ref}[4]$ & LIM & HAM & Ref [4] & LIM & HAM & $\operatorname{Ref}[4]$ \\
\hline 0.25 & $\begin{array}{l}1.0575 \\
\end{array}$ & $\overline{1.0575}$ & 1.0634 & 1.0509 & 1.0509 & 1.0535 & 1.0509 & 1.0509 & 1.0535 \\
\hline 0.5 & 1.2121 & 1.212 & 1.2388 & 1.1892 & 1.1891 & 1.2038 & 1.1892 & 1.1891 & 1.2038 \\
\hline 0.75 & 1.4308 & 1.4305 & 1.4832 & 1.3874 & 1.3872 & 1.4172 & 1.3874 & 1.3872 & 1.4172 \\
\hline 1 & 1.6886 & 1.6881 & 1.7679 & 1.6232 & 1.6227 & 1.6691 & 1.6232 & 1.6227 & 1.6691 \\
\hline 1.25 & 1.9703 & 1.9694 & & 1.8827 & 1.8819 & & 1.8827 & 1.8819 & \\
\hline 1.5 & 2.2671 & 2.2659 & 2.4000 & 2.1574 & 2.1563 & 2.2355 & 2.1574 & 2.1563 & 2.2355 \\
\hline 1.75 & 2.5739 & 2.5724 & & 2.4422 & 2.4408 & & 2.4422 & 2.4408 & \\
\hline 2 & 2.8876 & 2.8857 & 3.0729 & 2.7342 & 2.7325 & 2.8439 & 2.7342 & 2.7325 & 2.8439 \\
\hline
\end{tabular}

The ratio of linear to non-linear frequency for rectangular plates with aspect ratios of 2 and 4 are presented in tables 3 and 4 for different arrangements. The [90/0/0/90] arrangement shows the least frequency ratio. For all arrangements excluding the above-mentioned, the frequency ratio increases when aspect ratio increases. 
Table 3 Frequency ratio of composite rectangular plate with different arrangements using various methods $\left(E_{1} / E_{2}=40, G_{12} / E_{2}=0.5, v_{12}=0.25, a / b=2\right)$

\begin{tabular}{|c|c|c|c|c|c|c|c|c|c|}
\hline \multirow[t]{2}{*}{$W / h$} & \multicolumn{3}{|c|}{$\left[0^{\circ} / 90^{\circ} / 0^{\circ} / 90^{\circ}\right]$} & \multicolumn{3}{|c|}{$\left[0^{\circ} / 90^{\circ} / 90^{\circ} / 0^{\circ}\right]$} & \multicolumn{3}{|c|}{$\left[90^{\circ} / 0^{\circ} / 0^{\circ} / 90^{\circ}\right]$} \\
\hline & LIM & HAM & Ref [4] & LIM & HAM & $\operatorname{Ref}[4]$ & LIM & HAM & Ref [4] \\
\hline 0.25 & 1.0535 & 1.0522 & 1.0645 & 1.1297 & 1.1297 & 1.1327 & 1.0322 & 1.0322 & 1.0340 \\
\hline 0.5 & 1.2261 & 1.2156 & 1.2427 & 1.4461 & 1.4458 & 1.4674 & 1.1226 & 1.1226 & 1.1331 \\
\hline 0.75 & 1.4797 & 1.46 & 1.4905 & 1.8538 & 1.8531 & 1.8946 & 1.2577 & 1.2576 & 1.2798 \\
\hline 1 & 1.7704 & 1.7463 & 1.7787 & 2.3052 & 2.304 & 2.3652 & 1.4242 & 1.4239 & 1.4593 \\
\hline 1.25 & 2.0782 & 2.0531 & & 2.7795 & 2.7777 & & 1.6124 & 1.6119 & \\
\hline 1.5 & 2.3955 & 2.3703 & 2.4178 & 3.2666 & 3.2644 & 3.3634 & 1.8155 & 1.8148 & 1.8777 \\
\hline 1.75 & 2.7184 & 2.6935 & & 3.7619 & 3.7591 & & 2.0292 & 2.0283 & \\
\hline 2 & 3.045 & 3.0205 & 3.0976 & 4.2622 & 4.2591 & 4.3949 & 2.2505 & 2.2493 & 2.3396 \\
\hline
\end{tabular}

Table 4 Frequency ratio of composite rectangular plate with different arrangements using various methods $\left(E_{1} / E_{2}=40, G_{12} / E_{2}=0.5, v_{12}=0.25, a / b=4\right)$

\begin{tabular}{|c|c|c|c|c|c|c|c|c|c|}
\hline \multirow[t]{2}{*}{$W / h$} & \multicolumn{3}{|c|}{$\left[0^{\circ} / 90^{\circ} / 0^{\circ} / 90^{\circ}\right]$} & \multicolumn{3}{|c|}{$\left[0^{\circ} / 90^{\circ} / 90^{\circ} / 0^{\circ}\right]$} & \multicolumn{3}{|c|}{$\left[90^{\circ} / 0^{\circ} / 0^{\circ} / 90^{\circ}\right]$} \\
\hline & LIM & HAM & Ref [4] & LIM & HAM & $\operatorname{Ref}[4]$ & LIM & HAM & Ref [4] \\
\hline 0.25 & 1.0516 & 1.0499 & 1.0653 & 1.1657 & 1.1657 & 1.1707 & 1.0309 & 1.0309 & 1.0326 \\
\hline 0.5 & 1.2267 & 1.2129 & 1.2454 & 1.5547 & 1.5544 & 1.5838 & 1.1178 & 1.1178 & 1.1275 \\
\hline 0.75 & 1.4865 & 1.4607 & 1.4956 & 2.0412 & 2.0403 & 2.0951 & 1.248 & 1.248 & 1.2688 \\
\hline 1 & 1.7831 & 1.7522 & 1.7863 & 2.5709 & 2.5694 & 2.6489 & 1.4092 & 1.4089 & 1.4423 \\
\hline 1.25 & 2.0962 & 2.0641 & & 3.1223 & 3.1202 & & 1.5917 & 1.5913 & \\
\hline 1.5 & 2.4178 & 2.3861 & 2.4303 & 3.6857 & 3.683 & 3.8099 & 1.7891 & 1.7885 & 1.8479 \\
\hline 1.75 & 2.7444 & 2.7136 & & 4.2562 & 4.2531 & & 1.997 & 1.9962 & \\
\hline 2 & 3.0745 & 3.0444 & 3.1148 & 4.8316 & 4.8279 & 5.0011 & 2.2127 & 2.2115 & 2.2971 \\
\hline
\end{tabular}

Tables 5 to 7 summarize comparison results for different modulus ratios.

Table 5: Frequency ratio of composite rectangular plate with different arrangements using various methods $\left(E_{1} / E_{2}=10, G_{12} / E_{2}=0.5, v_{12}=0.25\right)$

\begin{tabular}{|c|c|c|c|c|c|c|c|c|c|}
\hline \multirow[t]{2}{*}{$W / h$} & \multicolumn{3}{|c|}{$\left[0^{\circ} / 90^{\circ} / 0^{\circ} / 90^{\circ}\right]$} & \multicolumn{3}{|c|}{$\left[0^{\circ} / 90^{\circ} / 90^{\circ} / 0^{\circ}\right]$} & \multicolumn{3}{|c|}{$\left[90^{\circ} / 0^{\circ} / 0^{\circ} / 90^{\circ}\right]$} \\
\hline & LIM & HAM & Ref [4] & LIM & HAM & $\operatorname{Ref}[4]$ & LIM & HAM & $\operatorname{Ref}[4]$ \\
\hline 0.25 & 1.0518 & 1.0518 & 1.0556 & 1.0478 & 1.0478 & 1.0498 & 1.0478 & 1.0478 & 1.0498 \\
\hline 0.5 & 1.1925 & 1.1924 & 1.2113 & 1.1784 & 1.1783 & 1.1907 & 1.1784 & 1.1783 & 1.1907 \\
\hline 0.75 & 1.3936 & 1.3933 & 1.4315 & 1.3666 & 1.3664 & 1.3922 & 1.3666 & 1.3664 & 1.3922 \\
\hline 1 & 1.6326 & 1.6321 & 1.6906 & 1.5918 & 1.5913 & 1.6314 & 1.5918 & 1.5913 & 1.6314 \\
\hline 1.25 & 1.8952 & 1.8944 & & 1.8403 & 1.8396 & & 1.8403 & 1.8396 & \\
\hline 1.5 & 2.1731 & 2.172 & 2.2715 & 2.1041 & 2.1031 & 2.1722 & 2.1041 & 2.1031 & 2.1722 \\
\hline 1.75 & 2.4612 & 2.4597 & & 2.3782 & 2.3769 & & 2.3782 & 2.3769 & \\
\hline 2 & 2.7562 & 2.7545 & 2.8941 & 2.6595 & 2.6578 & 2.7553 & 2.6595 & 2.6578 & 2.7553 \\
\hline
\end{tabular}

Obviously, there is a greater frequency ratio for higher modulus ratios; however symmetric $[90 / 0 / 0 / 90]$ shows an opposite behavior. 
Table 6 Frequency ratio of composite rectangular plate with different arrangements using various methods $\left(E_{1} / E_{2}=10, G_{12} / E_{2}=0.5, v_{12}=0.25, a / b=2\right)$

\begin{tabular}{|c|c|c|c|c|c|c|c|c|c|}
\hline \multirow[t]{2}{*}{$\overline{W / h}$} & \multicolumn{3}{|c|}{$\left[0^{\circ} / 90^{\circ} / 0^{\circ} / 90^{\circ}\right]$} & \multicolumn{3}{|c|}{$\left[0^{\circ} / 90^{\circ} / 90^{\circ} / 0^{\circ}\right]$} & \multicolumn{3}{|c|}{$\left[90^{\circ} / 0^{\circ} / 0^{\circ} / 90^{\circ}\right]$} \\
\hline & LIM & HAM & Ref [4] & LIM & HAM & Ref [4] & LIM & $\overline{\text { HAM }}$ & $\operatorname{Ref}[4]$ \\
\hline 0.25 & 1.0521 & $\overline{1.0513}$ & 1.0588 & 1.0991 & 1.0991 & 1.0986 & 1.0334 & 1.0334 & 1.0352 \\
\hline 0.5 & 1.2122 & 1.2059 & 1.2222 & 1.35 & 1.3498 & 1.3579 & 1.1271 & 1.1271 & 1.1375 \\
\hline 0.75 & 1.4472 & 1.4345 & 1.4516 & 1.684 & 1.6835 & 1.7013 & 1.2665 & 1.2664 & 1.2885 \\
\hline 1 & 1.7194 & 1.7034 & 1.7204 & 2.0611 & 2.0602 & 2.0877 & 1.438 & 1.4377 & 1.4729 \\
\hline 1.25 & 2.0104 & 1.993 & & 2.462 & 2.4606 & & 1.6313 & 1.6309 & \\
\hline 1.5 & 2.3119 & 2.294 & 2.3206 & 2.8769 & 2.875 & 2.9210 & 1.8396 & 1.839 & 1.6795 \\
\hline 1.75 & 2.6198 & 2.6018 & & 3.3004 & 3.2981 & & 2.0586 & 2.0576 & \\
\hline 2 & 2.9321 & 2.914 & 2.9620 & 3.7298 & 3.727 & 3.7908 & 2.285 & 2.2839 & 2.3730 \\
\hline
\end{tabular}

Table 7 Frequency ratio of composite rectangular plate with different arrangements using various methods $\left(E_{1} / E_{2}=10, G_{12} / E_{2}=0.5, v_{12}=0.25, a / b=4\right)$

\begin{tabular}{|c|c|c|c|c|c|c|c|c|c|}
\hline \multirow[t]{2}{*}{$W / h$} & \multicolumn{3}{|c|}{$\left[0^{\circ} / 90^{\circ} / 0^{\circ} / 90^{\circ}\right]$} & \multicolumn{3}{|c|}{$\left[0^{\circ} / 90^{\circ} / 90^{\circ} / 0^{\circ}\right]$} & \multicolumn{3}{|c|}{$\left[90^{\circ} / 0^{\circ} / 0^{\circ} / 90^{\circ}\right]$} \\
\hline & LIM & HAM & Ref [4] & LIM & HAM & Ref [4] & LIM & HAM & Ref [4] \\
\hline 0.25 & 1.0523 & & & 1.1236 & & & 1.0326 & 1.0326 & 1.0342 \\
\hline 0.5 & & & & & & & & & \\
\hline 0.75 & 4645 & & & & & & & & 11 \\
\hline 1 & $1.74^{\prime}$ & 1.7256 & 1.72 & 2 & 2. & 2.3117 & & & 1.4613 \\
\hline 1.25 & & 2.0 & & & & & & & \\
\hline 1.5 & & & 2.3 & 18 & & 3.2788 & 1.8 & & 1.8811 \\
\hline 1.75 & 2.674 & 2.6511 & & 3.6734 & 3.6709 & & 2.0388 & 2.0378 & \\
\hline 2 & 2.9941 & 2.9713 & 3.0204 & 4.1604 & 4.1573 & 4.2796 & 2.2618 & 2.2606 & 2.3444 \\
\hline
\end{tabular}

It can be concluded from data given in tables above that frequency ratio increases with an increase in aspect ratio and modulus ratio and decreases when number of layers increases. It should be noted that added number of layers will result in a raise of the frequency ratio.

\section{CONCLUSION}

In this study, a composite laminated plate model is established to verify the vibrational behaviors of composite plate. The von-Karman's assumption and efficient approximate method (LIM) is employed to derive the nonlinear governing equation of motion. Analytical expressions are presented for nonlinear natural vibration analysis of composite laminated plate. Comparing with other Results, it is shown that the approximate analytical solutions are in very good agreement with the corresponding solutions. The effects of different parameters such as layer arrangements, skew angles and aspect ratio on the natural frequencies of the composite laminated plates are also studied. Results show that for all arrangements but [90/0/0/90], the frequency ratio increases when aspect ratio increases. The frequency ratio increases with an increase in aspect ratio and modulus ratio and decreases when number of layers increases. Furthermore, the symmetrical arrangement plates have the same frequency ratios values. 


\section{References}

[1] Chu HN, Herrmann G. Plate Influence of large amplitudes on free flexural vibrations of rectangular plates. ASME J Appl Mech 1956;23:532-540.

[2] Singha MK, Daripa R. Nonlinear vibration of symmetrically laminated composite skew plates by finite element method. International Journal of Non-Linear Mechanics 2007;42(9)1144-1152.

[3] Raju IS, Rao GV, Raju KK. Effect of longitudinal or inplane deformation and inertia on the large amplitude flexural vibrations of slender beams and thin plates. Journal of Sound and Vibration 1976;49(3):415422 .

[4] Singh S, Raju KK, Rao GV. Non-linear vibrations of simply supported rectangular cross-ply plates. Journal of Sound and Vibration 1990;142(2):213-226.

[5] Singha MK, Ganapathi M. Large amplitude free flexural vibrations of laminated composite skew plates. International Journal of Non-Linear Mechanics.2004;39(10):1709-1720.

[6] Malekzadeha P, Karami G. Differential quadrature nonlinear analysis of skew composite plates based on FSDT. Engineering Structures 2006;28(9)p1307-1318.

[7] Pirbodaghi T, Ahmadian MT, Fesanghary M. On the homotopy analysis method for nonlinear vibration of beams. Mechanics Research Communications 2009;36(2):143-148.

[8] Younesian D, Askari H, Saadatnia Z, KalamiYazdi M. Frequency analysis of strongly nonlinear generalized Duffing oscillators using He's frequency amplitude formulation and He's energy balance method. Comput Math Appl 2010;59:3222-3228.

[9] Yue YS, Lu FM. The max_min approach to a relativistic equation. Computers and Mathematics with Applications 2009;58: 2131_2133.

[10] He JH. Homotopy perturbation method for solving boundary value problems. Phys Lett 2006;A 350:87-88.

[11] He JH. Some asymptotic methods for strongly nonlinear equations. International Journal of Modern Physics B 2006;20(10):1141-1199.

[12] Rafieipour H,Lotfavar A, Mansoori MH. New analytical approach to nonlinear behavior study of asymmetrically LCBs on nonlinear elastic foundation under Steady axial and thermal loading. Latin American Journal of Solids and Structures 2012;9(5):531-545.

[13] Reddy J. Mechanics of Laminated Composite Plates Theory and Analysis: CRC, Boca Raton, 1997.

[14] Rao SS. Vibration of Continuous Systems: John Wiely \& Sons, New Jersy, 2007.

[15] Hesameddini E, Latifizadeh H. Reconstruction of Variational Iteration Algorithms using the Laplace Transform. Int J Nonlinear Sci Numer Simul 2009;10(10):1365-1370. 


\section{Appendix}

$\mathrm{I}_{i}$ coefficients in the second approximation of deflection.

$$
\begin{aligned}
& \mathrm{I}_{0}=990904320 \mathrm{~A}\left(\left(\Pi_{1}+\frac{21}{16} \Pi_{3}-2 \Pi_{4}\right) \mathrm{A}^{2}+\left(-\frac{1}{32} \Pi_{3}^{2}+\left(-\frac{49}{512} \Pi_{5}+\frac{41}{48} \Pi_{4}\right) \Pi_{3}-\frac{2}{3} \Pi_{4}^{2}\right) \mathrm{A}+\left(\frac{13}{4096} \Pi_{5}^{2}+\frac{31}{72} \Pi_{4}^{2}\right) \Pi_{3}-\frac{23}{36} \Pi_{4}^{3}\right) \\
& \mathrm{I}_{1}=1981808640 \mathrm{~A}^{4}+\left(-54190080 \Pi_{5}-1052835840 \Pi_{3}-1101004800 \Pi_{1}-7741440 \Pi_{2}+1761607680 \Pi_{4}\right) \mathrm{A}^{3} \\
& +\left(26512128 \Pi_{3}^{2}+\left(-595574784 \Pi_{4}+101007360 \Pi_{5}\right) \Pi_{3}-7741440 \Pi_{5}^{2}+399114240 \Pi_{4}^{2}\right) \mathrm{A}^{2}+ \\
& \left(\left(-457900032 \Pi_{4}^{2}-3740640 \Pi_{5}^{2}\right) \Pi_{3}+690880512 \Pi_{4}^{3}+574560 \Pi_{5}^{3}\right) \mathrm{A}-14364 \Pi_{5}^{4}
\end{aligned}
$$$$
\mathrm{I}_{2}=110100480 \mathrm{~A}\left(\left(\Pi_{1}-3 \Pi_{3}+2 \Pi_{4}\right) \mathrm{A}^{2}+\left(-\frac{1}{64} \Pi_{3}^{2}+\left(-2 \Pi_{4}-\frac{51}{1024} \Pi_{5}\right) \Pi_{3}+2 \Pi_{4}^{2}\right) \mathrm{A}+\left(\frac{7}{16} \Pi_{4}^{2}+\frac{3}{512} \Pi_{5}^{2}\right) \Pi_{3}-\frac{2}{3} \Pi_{4}^{3}\right)
$$$$
\mathrm{I}_{3}=\left(7741440 \Pi_{2}+54190080 \Pi_{5}+61931520 \Pi_{3}\right) \mathrm{A}^{3}+\left(\begin{array}{l}
8547840 \Pi_{3}^{2}+\left(3870720 \Pi_{5}-45158400 \Pi_{4}\right) \Pi_{3} \\
+5806080 \Pi_{5}^{2}+41287680 \Pi_{4}^{2}
\end{array}\right) \mathrm{A}^{2}
$$$$
+\left(\left(-181440 \Pi_{5}^{2}-16629760 \Pi_{4}^{2}\right) \Pi_{3}-544320 \Pi_{5}^{3}+13762560 \Pi_{4}^{3}\right) \mathrm{A}+15120 \Pi_{5}^{4}
$$$$
\mathrm{I}_{4}=20643840 \mathrm{~A}\left(\mathrm{~A}^{2} \Pi_{3}-\frac{1}{10} \Pi_{3}\left(\frac{49}{16} \Pi_{5}-6 \Pi_{4}+\Pi_{3}\right) \mathrm{A}+\left(-\frac{2}{45} \Pi_{4}^{2}+\frac{3}{320} \Pi_{5}^{2}\right) \Pi_{3}+\frac{4}{45} \Pi_{4}^{3}\right)
$$$$
\mathrm{I}_{5}=-483840 \mathrm{~A}\left(\left(\Pi_{3}^{2}+\left(-\frac{8}{3} \Pi_{5}-\frac{40}{9} \Pi_{4}\right) \Pi_{3}-4 \Pi_{5}^{2}\right) \mathrm{A}+\left(-\frac{32}{27} \Pi_{4}^{2}+\frac{\Pi_{5}^{2}}{24}\right) \Pi_{3}+\frac{\Pi_{5}^{3}}{8}\right)
$$$$
\mathrm{I}_{6}=147456 \mathrm{~A} \Pi_{3}\left(\left(\Pi_{3}+\frac{51}{16} \Pi_{5}\right) \mathrm{A}-\frac{3}{8} \Pi_{5}^{2}+\frac{4}{9} \Pi_{4}^{2}\right)
$$

$$
\mathrm{I}_{7}=26880 \Pi_{3}^{2} \mathrm{~A}^{2}+10080 \Pi_{5}^{2}\left(3 \Pi_{5}+\Pi_{3}\right) \mathrm{A}-945 \Pi_{5}^{4} \quad \mathrm{I}_{8}=3840 \Pi_{5}^{2} \mathrm{~A} \Pi_{3} \quad \mathrm{I}_{9}=189 \Pi_{5}^{4}
$$

where

$$
\Pi_{1}=\frac{\alpha_{1} A^{2} \alpha_{2}}{\omega^{4}}, \quad \Pi_{2}=\frac{\alpha_{1} A^{3} \alpha_{3}}{\omega^{4}}, \quad \Pi_{3}=\frac{\alpha_{2} A^{4} \alpha_{3}}{\omega^{4}}, \quad \Pi_{4}=\frac{\alpha_{2} A^{2}}{\omega^{2}}, \quad \Pi_{5}=\frac{\alpha_{3} A^{3}}{\omega^{2}}
$$

